\title{
Ethical tensions associated with the promotion of public health policy in health visiting: a qualitative investigation of health visitors' views
}

\author{
Julie Catherine Greenway ${ }^{1,2}$, Vikki Ann Entwistle ${ }^{3}$ and Ruud terMeulen ${ }^{4,5}$ \\ ${ }^{1} \mathrm{PhD}$ Student, Centre for Ethics in Medicine, University of Bristol, Bristol, UK \\ ${ }^{2}$ Health Visitor, Worcestershire Health and Care NHS Trust, Worcs, UK \\ ${ }^{3}$ Professor of Values in Health Care, Social Dimensions of Health Institute, Dundee, UK \\ ${ }^{4}$ Professor of Ethics in Medicine, University of Bristol, Bristol, UK \\ ${ }^{5}$ Director of the Centre for Ethics in Medicine, School of Social and Community Medicine, University of Bristol, \\ Bristol, UK
}

\begin{abstract}
Aim: To explore whether and how health visitors experience ethical tensions between the public health agenda and the need to be responsive to individual clients. Background: Current health policy in England gives health visitors a key role in implementing the government's public health agenda. Health visitors are also required by their Professional Code to respond to the health-related concerns and preferences of their individual clients. This may generate tensions. Methods: A total of 17 semistructured individual interviews covering participants' experiences of implementing public health interventions and perceptions of the ethical tensions involved were conducted. Interviews were audio-recorded, transcribed and analysed thematically using a Framework approach. Findings: Health visitors raised a number of ethical concerns, which they attributed to organisational resource allocation and the introduction of protocols and targets relating to public health goals. They did not always regard it as appropriate to raise topics that employing organisations had identified as public health priorities with particular clients for whom they were not priorities, or who had other more pressing needs. They noted that resources that were allocated towards reaching public health targets were unavailable for clients who needed support in other areas. Organisational protocols designed to monitor performance put pressure on health visitors to prioritise achieving targets and undermined their ability to exercise professional judgement when supporting individual clients. This had implications for health visitors' sense of professionalism. Health visitors saw trusting relationships as key to effective health visiting practice, but the requirement to implement public health priorities, combined with a lack of resources in health visiting, eroded their ability to form these. Policies need to be evaluated with regard to their impact upon a broader range of processes and outcomes than public health goals. The erosion of health visitors' professional values and ability to develop relationships with clients could have numerous adverse implications.
\end{abstract}

Key words: community health nursing; ethics; professional-patient relations; public health

Received 23 October 2011; revised 20 July 2012; accepted 22 July 2012;

first published online 10 August 2012

Correspondence to: Julie C. Greenway, Bewdley Medical Practice,

Dog Lane, Bewdley, Worcs DY12 2EG, UK. Email: julie.

greenway@blueyonder.co.uk or julie.greenway@hacw.nhs.uk

(C) Cambridge University Press 2012 


\section{Background}

The question of whether and how health visitors experience ethical tensions between obligations to promote a public health agenda and to be responsive to individual clients arises against a backdrop of changes in health visiting policy and practice. A brief overview of and summary of current policy is provided here. Successive government policy documents in England have identified health visitors as having a key role in implementing public health agendas (Department of Health, 1999a; 1999b; 2000; 2001; 2002; 2004; $2006 ; 2007 ; 2010 a ; 2011)$. Health visitors' work has always incorporated a public or population health element, but this has been explicitly emphasised in recent years and a number of health issues that health visitors address, including breastfeeding and smoking cessation, have been identified as national priorities. For some health issues, primary care organisations are given targets to reach, for example the percentage of babies who are breastfed to at least six weeks. In response, these organisations adopt various strategies to encourage their staff to work towards these targets, and to ensure they collect data to document their achievement.

However, health visitors work with clients on an individual basis and are also required to abide by their Nursing and Midwifery Council (NMC) Code. The Code tells them 'you must listen to the people in your care and respond to their concerns and preferences' (NMC, 2007: 3). A professional obligation to respect individual clients' preferences (often associated with respecting their personal autonomy) might not be compatible with some approaches to promoting public health targets. As Cowley and Frost (2006) have noted, health visitors may experience ethical dilemmas as they are required to both respect clients as individuals and to protect and support the health of the wider community.

A number of other changes in the organisation of health visiting work are associated with the shift towards a public health agenda and efforts to improve service efficiency. These include efforts to target resources preferentially to socioeconomically disadvantaged areas, a shift away from home-based towards clinic-based health care provision, and a shift away from individual caseload working to corporate caseload working.
A number of empirical research studies have led to a richer understanding of some of the important ethical issues and concerns faced by nurses with public health roles in their day-to-day practice (Beidler, 2005; Clancy and Svensson, 2007; Moules et al., 2010). Although some of these studies have been conducted outside of the United Kingdom and among health professionals with different titles, some of these issues and concerns may also apply to health visiting in England. Calls have also been made to pay more attention to the institutional processes that shape and constrain ethical dialogue and practice (Austin, 2007).

Potential tensions between concerns to respect personal autonomy and efforts to promote the health of the wider population are important issues in a developing literature on public health ethics (Bayer et al., 2007; Dawson and Verweij, 2007; Holland, 2007; Calman, 2009). Renewed interest in public health led to awareness that individually oriented medical/nursing ethical frameworks can be inappropriate when a population-based perspective is adopted and emphasis placed on disease prevention rather than treatment (Kass, 2001; Callahan and Jennings, 2002; Bayer and Fairchild, 2004). A number of theoretical and conceptual models/frameworks have subsequently been developed to help public health professionals consider the ethical implications of their work. Kass (2001) proposed a six-step framework or analytic tool to help public health professionals consider the ethical implications of proposed interventions, policy proposals, research initiatives and programmes. Childress et al. (2002) discerned a set of 'general moral considerations' relevant to public health. These include producing benefits, avoiding harms, distributing benefits and burdens fairly, respecting autonomous choices, protecting privacy and confidentiality and building and maintaining trust. In 2007, the Nuffield Council on Bioethics presented their stewardship model. They proposed an 'intervention ladder' with a range of interventions across a spectrum, from the least to the most coercive, which can be used to attempt to change the behaviour of individuals and communities in order to improve population health. Tannahill (2008) developed a decisionmaking triangle that encourages the explicit application of an identified set of ethical principles and the use of available evidence and theory to inform judgements. 
As recent government policy documents (Department of Health, 2007; 2010a; 2011) seek to involve health visitors more explicitly in efforts to improve population health through changing individual's health behaviour, there is the potential for ethical tensions to be exacerbated.

As plans are underway to expand and strengthen health visiting services (Department of Health, 2011), attention to this issue becomes more pressing. The recent Health Visitor Implementation Plan outlines a plan to have 4200 more health visitors in post by 2015 by expanding the number of training places available for new recruits and by offering return-to-practice opportunities to enable qualified health visitors who have been out of practice to refresh and develop their skills.

\section{Aims}

The work reported here was part of a larger project that aimed to investigate:

1) How health visitors experience, perceive and manage any ethical tensions arising from aspects of their work relating to the pursuit of public health goals in the context of care for individual clients

2) How well their professional education equips them to deal with ethical tensions associated with this role.

This paper focuses on findings relating to the first aim. It explores the feelings of tension, concern or discomfort, which health visitors experience and associate with issues of what is right/wrong, good/ bad, appropriate/inappropriate in their work, for example as they strive to meet multiple demands or have to juggle conflicting priorities.

\section{Design}

Exploratory cross-sectional qualitative (interpretive) study using semi-structured individual interviews with health visitors.

\section{Methods}

\section{Sampling, recruitment and consent}

To help ensure diversity within the sample, health visitors were recruited from two English
Primary Care Trusts (PCTs - the main primary care provider organisations). One PCT covered a large city, whereas the other covered a smaller city, a number of towns and rural areas. The latter PCT had been established through the amalgamation of three smaller PCTs in 2006. The researchers sought to include health visitors from different backgrounds and with varying levels of experience, but were reliant for this on a range of individual health visitors opting into the study.

Recruitment was conducted via health visitors' monthly professional meetings, which were held in both PCTs involved in the study. The PCT based in the large city held a central professional meeting for all the health visitors employed by the Trust. The other PCT that covered a wider geographical area held three separate professional meetings based in different locations. Information about the study was therefore presented at four health visitors' monthly professional meetings where participant information booklets and reply forms and stamped addressed envelopes were provided for those interested in participating. The information explained that the interviewer was a health visitor.

Appointments were arranged to discuss the study in more detail with individuals who expressed interest. They took place at health visitors' work bases (GP surgeries, clinics or health centres). Individuals who were willing to be interviewed after this discussion signed a pre-interview consent form. At the end of each interview, the participants were asked to sign a post-interview consent form to indicate consent to the research team publishing anonymous quotations from the interview. All participant forms were stored in a locked draw in a university office.

Approval to conduct the research was obtained from the National Health Service (NHS) National Research Ethics Service and relevant NHS Research and Development offices.

\section{Data collection}

Interviews were conducted at participants' work bases. They varied in length but averaged around $45 \mathrm{~min}$. Topic guides were used to help ensure coverage of key topics that our reading of relevant literature and awareness of potential concerns suggested were relevant to the research aims. The guides listed topics to be explored via 
open, non-leading questions, which established the issue to be discussed but avoided suggesting particular responses.

Three pilot interviews were initially carried out and reviewed by the team to confirm that the topic guides and interview approach would yield useful, relevant data.

The topics included participants':

- Perceptions of their public health role and examples of public health interventions that they carry out

- Experiences of implementing public health interventions

- Perceptions of any ethical tensions in implementing public health interventions.

The researchers used a broad working definition of public health interventions, including any programmes or activities, which had the aim of promoting and protecting the health of a population.

All interviews were audio-recorded. A total of 17 semi-structured interviews with health visitors were carried out between March and August 2008. Four participants were recruited following presentation of the research at the professional meeting in the large city; five were recruited following presentation of the research in the smaller city; six were recruited following presentation in the towns and two from the professional meeting in the rural areas. All those who returned the reply forms expressing an interest in taking part in the study were interviewed. Because a good range of health visitors were included in these samples and because similar issues were recurring and no new issues were emerging in the more recent interviews, the research team agreed that no further recruitment was needed.

\section{Data analysis}

Interviews were transcribed verbatim and personal identifiers removed. The matrix-based Framework approach to qualitative data analysis was used to facilitate rigorous and transparent management of data (Ritchie and Spencer, 1994). Transcripts were entered into Atlas.ti software to facilitate data management. Interview transcripts were read repeatedly for initial familiarisation and to identify key ideas and recurring themes. A thematic coding framework was then devised to cover both issues of prior interest (which had featured in the topic guides) and issues raised by participants. Twelve broad thematic domains were identified. Atlas.ti was used to apply the codes for these thematic domains systematically to each transcript, and thematic charts were produced to summarise the data coded against each domain heading, and to further organise these data within the broad thematic domains. Each participant was allocated a row in the matrix and separate columns were used for each thematic domain then sub-domain. Summary entries reflected participants' own words and noted links back to transcript pages to facilitate revisiting of data and retrieval of quotations that could illustrate key points. The chart-based approach facilitated consideration of the range of reported experiences and reviews within thematic domain and sub-domain. Whole transcripts were re-read during the analysis to ensure ongoing sensitivity to subtle issues and context.

Analysis was led by J.G., but in order to ensure quality co-authors read transcripts, discussed the interpretation of data and the development of codes and checked coding and charting. This scrutiny of interviews and key analytic steps by the whole research team was an important way of ensuring that leading questions had been avoided or were recognised during the analysis, and that interpretations were robust.

In the illustrative quotations presented below, the health visitors have been assigned study identifiers HV 01-HV 17.

\section{Findings}

All 17 health visitor participants were female. They represented a cross-section of ages and had been in practice for various lengths of time. Nine had been qualified between one and ten years; five had been qualified between 10 and 20 years and three had been qualified between 20 and 30 years. They had varying caseload numbers, worked in both affluent and less affluent areas and engaged with clients who represented a wide range of socioeconomic groupings and national/ ethnic backgrounds including British, Polish, African, Pakistani, Bangladeshi and Somali. Some health visitors worked as part of a team but maintained their own identifiable caseload, some worked 
corporately on large combined caseloads and others worked alone on their own caseload.

Participants described a variety of work situations in which they had experienced some form of ethical tension or concern. Most of these were associated with organisational efforts to pursue a public health agenda and were further compounded by the way their work was organised and by limited health visiting resources.

The two PCTs from which health visitors were recruited were both working to public health targets relating to breastfeeding and smoking cessation. Participants often reported that they were required by local protocols to raise these issues with clients and to collect data about clients' behaviour for the purpose of performance monitoring against these targets. Some also noted that performance monitoring was linked to their own individual performance rather than that of a group or the organisation more generally.

Organisational arrangements such as corporate caseload working and moves to clinic rather than home-based care provision were also cited by a number of participants as root causes of a number of the tensions they experienced. The general lack of resources within health visiting was reported to have resulted in increased workloads as a result of losing health visitor posts. Participants reported having insufficient time to carry out routine antenatal contacts or to carry out home visits to new parents as frequently as they would have liked in the first six weeks after the baby's birth. They identified this as being a key time for establishing and building up a relationship with their clients and reported that home visiting enabled clients to raise issues in a way that couldn't always be achieved in the clinic setting.

In the sections that follow, we consider the ethical tensions raised by the participants within three main themes: differing priorities; health visitors' professionalism; and health visitor-client relationships.

\section{Differing priorities}

Health visitors stressed the importance of assessing individual clients' health needs and individual clients' perceived priorities for the provision of appropriate health visiting support. They also stressed that the effectiveness and appropriateness of any particular intervention could vary across clients and depend strongly on their particular situations, personal/cultural beliefs, attitudes and priorities.

Health visitors commented on a number of situations in which they thought that health topics and interventions that were designated public health priorities and that they were encouraged by organisational policies to deliver were not always priorities for their particular clients - at least not at that time. For example, health visitors reported that the requirement to offer smoking cessation advice was inappropriate for some clients for whom smoking might be an important coping mechanism for their current highly stressful situations:

...if they're in a grotty flat on a top floor and clearly just surviving day to day... smoking is not the thing that they're going to stop because it's probably keeping them sane, sadly.

(HV 11)

I've been reading ... that the highest group of smokers now is young mothers ... and we could actually be causing more harm than good...it could be that if mum does give up smoking, she's stressed...she takes it out on the children so the best thing could be for her to smoke.

(HV 09)

As well as the need for interventions to be appropriate for particular clients, participants viewed timing as an important consideration relating to the likely effectiveness of interventions intended to promote lifestyle changes such as giving up smoking:

...unless they're ready to make that change it's not going to be effective... you can tell them the information and hopefully if they want to make that change they will come to you for support when they want to make that change.

(HV 09)

The organisationally imposed need to raise certain public health priority topics during initial visits to particular clients was reported to be inappropriate in some circumstances, particularly when the topics were sensitive and they had not yet established a rapport with those clients:

I don't always feel comfortable asking them about the smoking...especially because 
you're expected to do it on the primary visit or the first few visits when you've met people and it always seems a bit intrusive to me when you're trying to build a rapport.

(HV 02)

One way the participants managed the discomfort of collecting data during initial visits was to distance themselves from the requirement to gather this information by informing clients that they needed to gather the information for their employers:

I think it's perhaps...almost made easier at the moment because as professionals we can all say "...we've been asked to gather this information."”

(HV 06)

...so I just bring it up and say "well another thing that they'd like us to talk about at this time is..."

(HV 07)

Rather than adhering unquestioningly to the organisational priorities, a number of participants reported considering their clients' own perceptions regarding their health priorities:

I hope what we do as health visitors, is...to look at it through the families' perception$s$... and I think that's probably why smoking doesn't really kind of develop high on our radar. Because for a lot of families we can say, "Oh have you ever thought about quitting?" "Oh no, no I can't do that...I'm too stressed at the moment." And it's just not their priority.

(HV 16)

I think you have to weigh up their lifestyle and what's important to them.

(HV 01)

The difficulties experienced by health visitors as they try to weigh up the competing priorities and interests of their employer, their clients' perceptions of their health needs and their own assessment of their clients' health needs was summarised by one participant:

...we're in such a difficult situation, because we're looking at our perceived health issues of that family, trying to ascertain what the family's perceptions of their health needs are, and what does the PCT suggest that these health needs are? ... you're so kind of encompassed with it all, you're going to just look at your own perceptions really and the family's, and try and focus on those. But there is huge pressure from everywhere.

(HV 16)

A second, linked, priority-related tension occurred when health visitors recognised that clients had strong needs that were not prioritised by public health-oriented organisational strategies:

...we're looking to try and meet the needs of the families here, which often doesn't reflect those needs that are identified by X(PCT) ...there is a high percentage of drug use and alcohol use, and really that's the kind of focus we want to prevent people from entering, because then it just develops into domestic abuse, and we've got the child protection difficulties as a result...there are often other issues... trying to get these children out to the groups, trying to improve their behaviour, their speech. Housing is a huge issue for a lot of these families... huge amounts of depression...

(HV 16)

Participants identified a number of reasons why these clients' needs could be neglected. The requirement to raise some topics for data-gathering purposes could crowd out the agenda and tend to lead health visitors neglecting other topics:

...alcohol consumption...that is something that perhaps I don't talk that much about which is probably...something I should be, sort of in the middle class area...it's something I perhaps don't touch on at all and I should.

Some participants suggested this tendency could be reinforced because they were given less information and training relating to topics that were not organisational public health priorities, and so were less confident about raising these topics:

I wouldn't feel as comfortable talking about alcohol consumption with families because I haven't been given much information on that, I'm not sure of the government position... what the recommendations are... so I would 
find bringing that subject up far harder than smoking.

With regard to the distribution of resources, a number of participants expressed concern that when resources were directed towards attaining public health targets, clients who could benefit from support in non-prioritised areas missed out:

Breastfeeding, breastfeeding and breastfeeding...I think it's fantastic...I think it's great but I would like to see some of that resource perhaps shared with things like accident prevention... breastfeeding is very much the topic in this trust... a lot of resources go to breastfeeding.

(HV 13)

Several participants reported having been instructed to stop carrying out certain health promotion activities such as men's health days and work with travelling families as they no longer aligned with their employing organisation's priorities. They considered that this again led to neglect of the health needs of these non-prioritised groups.

Although one of the aims of resource prioritisation is to reduce socioeconomic inequalities, the fact that targets might be more readily met by concentrating efforts in affluent areas could go against this aim. When considering breastfeeding targets, it was thought to be easier to increase breastfeeding rates by concentrating efforts in affluent areas rather than in socioeconomically deprived areas where changing cultural norms could take a long time:

...you could increase your breastfeeding rates in quite affluent areas like $X$...that would be quite easy to increase. But to actually increase rates in an area like this, you're not going to see them in a year's time, and they want to see the changes now.

(HV 16)

\section{Health visitors' sense of professionalism}

Rather than being allowed to exercise their own professional judgement with regard to which health topics to address, health visitors reported that some topics were emphasised in ways that were unhelpful for particular people and were sometimes at variance with their own professional assessments of those particular clients' health needs.
In some cases, working in accordance with their employing organisation's protocols was reported to be little more than a 'tick box' exercise and a waste of time when the topics seemed inappropriate for a particular client:

...sometimes I think I talk about some things and I think, "Why am I wasting my time on this? Because I know they don't want to do it." I'm simply ticking the box...it doesn't mean you've discussed it in any sort of meaningful way. And I think it's often much more valuable when you go in and you start talking about something... important for them...if a topic comes up that is towards health and... is of benefit to that family, and what they want to talk about, then it's better to go along that line than just to be mindlessly ticking the boxes.

(HV 12)

Some participants expressed concern that if individual health visitors' names were linked to documented breastfeeding rates among women on their caseloads, their performance as health professionals might be inappropriately judged (for example, if low breastfeeding rates were interpreted as being purely the result of health visitors giving poor breastfeeding advice and support and high breastfeeding rates were attributed to effective health visiting interventions, and broader social influences on breastfeeding were not considered):

...we were talking about breastfeeding statistics, and one health visitor from a very nice area said, "Well my statistics are 96\%," ... and I thought, well you just can't compare... that doesn't mean anything does it? ...those women would breastfeed anyway, with or without your support ...but to even get women in a bottle-feeding culture thinking about breastfeeding, knowing a little bit more, is good. So I think we need to be careful about...the statistics.

(HV 14)

One participant hoped that such possibilities would not lead to inappropriate changes in the way health visitors promoted breastfeeding:

...our targets actually have changed and they will be looking at six week rates ... so I 
suppose that could affect how we push breastfeeding. I hope it doesn't. Previously they were just looking at initiation but now we're looking at six week targets for breastfeeding... they're going to have our names against those rates where previously it was the midwives.

(HV 09)

\section{Health visitor-client relationships}

A number of participants identified the health visitor-client relationship as being of key importance and necessary in order to deal with some of the competing priorities appropriately. The relationship was felt to be the key factor influencing health visitors' ability to raise health promotion topics with their clients and to support them through changes in health behaviour:

I think if... I've built up a trusting relationship with a family it's very much easier to approach any of these subjects. I think if you're meeting people...on a one-off occasion in clinic, it's a complete and utter waste of time...you've got to build up a relationship before you can support them through change.

(HV 13)

Some participants reported that they would only raise some sensitive health promotion topics with their clients if they knew them well and that they would be cautious in raising topics if it was the first time they had met them as they did not want to jeopardise the development of a good relationship with the client. They spoke of the importance of developing, maintaining and protecting this relationship so that they might be able to address other issues that were currently present or which may emerge at a later date:

...you need to try and keep the relationship with families and I think there's a time and place for raising public health issues and I think it depends on how comfortable you feel and whether you know them well enough to tackle that issue. You have to be very careful how you approach people because they won't open the door to you next time and if you do go in and identify an issue then you really need for that door to be left open so that you can go back in.

(HV 05)
Another reason given for the importance of developing the relationship was because of the recognition that behaviour change could be encouraged over a period of time. A number of participants used phrases such as 'planting a seed', a 'drip by drip' process or 'chipping away':

... you're drip-feeding these things in over a period of time... but I think that that is all then through building up the relationship with people.

...sometimes you can lead up to something so you can start and if you like plant a seed but then maybe it'll be two or three weeks before you go back and actually introduce the topic itself.

You have to sort of look at what's going to make the biggest impact for that family and try and chip away and promote healthy living.

(HV 01)

Although developing trusting relationships was seen as key for effective health visiting practice, the requirement to implement public health priorities combined with a lack of resources and some re-organised ways of working contributed to an erosion of the ability of health visitors to form these.

\section{Discussion}

The increasing emphasis on attaining public heath goals is creating a number of ethical tensions for health visitors. Being required to work towards meeting their employing organisation's prioritised public health goals often went against the grain of health visitors' broader professional view of their roles in assessing and responding to a range of health needs, taking into account their clients' particular situations and their own perceptions of their clients' priorities. The public health targets that had been set for populations sometimes clashed with health visitors' desires to effect the interventions they considered most applicable to the individual clients to whom they had a professional duty of care. This tension could be exacerbated when clients were experiencing crises such as those resulting 
from material disadvantage. Health visitors were aware that changing health behaviours such as smoking might not be a priority for particular clients, especially at particular times - smoking might, for example, help clients cope with current stressful situations. Although it is known that much ill-health occurs as a result of material disadvantage (Rogers, 2004), current policies still emphasise individuals' decisions regarding their health behaviours and tend to neglect the wider issues which affect health.

Working in accordance with organisational protocols also raised a number of concerns, particularly when the protocols required health visitors to collect data about clients' behaviours before they had established relationships with these clients, or when they seemed inappropriate for particular clients. Some health visitors adopted a strategy of distancing themselves from requirements they considered inappropriate for individual clients by informing clients that the requirements were externally imposed (for example, that information was being gathered for their employers). Having to collect data according to a pre-determined schedule and to raise topics that were felt to be inappropriate for certain clients was also felt by some participants to have a detrimental effect on their ability to develop relationships with those clients and this might impair the ability of health visitors to support clients with future needs.

If organisations put too strong an emphasis on reaching a particular set of public health goals, the key needs of some clients may remain unmet if health visitors fail to raise questions about and recognise them, or are unable to access resources to address them. Participants reported that they had less opportunity to gain knowledge and skills in non-prioritised topic areas such as alcohol consumption. This finding was similar to that from another recent study, which reported that although there is increasing concern about alcohol misuse health visitors reported that they were often unsure as to how to raise the issue and felt that they lacked the knowledge and skills to do this effectively (Lacey, 2009). The allocation of resources at the client level is an important component of the ethical issue of distributive justice. Client groups whose needs do not cohere with the prioritised topics may fail to receive much needed services if resources continue to be diverted away from work such as accident prevention, men's health and work with travelling families.

Although public health policy aims to reduce health inequalities between socioeconomic groups, working towards public health targets may actually result in a widening of the health inequalities gap. This is because participants reported that with regard to meeting short-term organisational breastfeeding targets, directing resources to the more affluent client groups who were thought to be more likely to respond to health promotion messages might result in practitioners meeting targets more easily.

The requirement to raise health topics according to organisational protocols may also have implications for health visitors' sense of professionalism. Although health visitors may make a professional judgement that certain topics are inappropriate for particular clients, they may be required by their employing organisations to raise these topics in order to record data for the purpose of target achievement and performance monitoring. Protocols designed to monitor performance relating to standardised targets may also have implications for health visitors' sense of professionalism when linked to individual rather than organisational performance.

Although health visitors in this study hoped that having their individual names against breastfeeding rates would not affect the way they promoted breastfeeding, working to attain performance targets may result in health professionals giving their clients insufficient information regarding alternative options. A recent systematic literature review carried out by Lakshman et al. (2009) found that mothers who used infant formula were not receiving sufficient information from health professionals about bottle-feeding and not feeling empowered to make decisions regarding their method of infant feeding, suggesting that the concerns of the health visitors in this study may have been justified. More generally, although audits based on the recommendations of guidelines might be performed to enable practitioners to evaluate their own practice, Rogers (2002) suggests that such auditing could lead to coercion on the part of health practitioners to ensure compliance with recommendations and meeting of targets with little regard for client preferences. Cribb (2005) also suggests that tightly defined budgets, guidelines, protocols, published performance indicators and financial 
incentive schemes can interfere with health professionals' relationships with their clients and undermine the autonomy of the health care professional to decide what is best for the client.

The development of a professional trusting relationship with clients was considered by a number of participants to be the key factor in knowing how and when to pursue topics of public health in work with particular clients. A number of other studies have demonstrated the importance of health visitors developing trusting relationships with their clients. De La Cuesta (1994) suggested that it was the trusting health visitor-client relationship that served to mediate between the policy agenda and the client's agenda. McNaughton (2000) found that building and maintaining relationships with clients was the central focus of home visiting and provided a foundation for problem identification and problem solving. If trusting relationships were not established, clients were likely to remain passive during home visits, avoid working with practitioners to promote family health and even refuse to allow practitioners' future access into their homes. Worth and Hogg (2000) concluded that the basis of effective interventions was the strength of the relationship between the health visitor and the parents and that in situations where parents reported having a poor relationship with their health visitor this may be related to parents having received few home visits or to parents having seen different health visitors and not being able to develop a relationship with a particular health visitor. In their research into health visiting assessment processes, Appleton and Cowley (2008a) also found that clients needed to build up a trusting relationship with their health visitor before they felt able to talk about their family's health issues. In a subsequent paper, Appleton and Cowley ( 2008b) develop the notion of 'gauging', which is about recognising that parents may not be able or ready to address needs that are evident to the health professional. They suggest that gauging encompasses an element of 'treading carefully' and that this skill is an important component of successful relationship building. It was particularly emphasised by health visitors in their study who did not know their clients well. Russell (2008) found that clients prefer to receive parenting advice and support from a health visitor with whom they have built a relationship.
The above studies tend to emphasise the instrumental value of developing and maintaining trust as a means of achieving other health-related goals, but developing and maintaining trust also has an important intrinsic value within good caring relationships (Held, 2006). Despite the importance attached to having good relationships with clients, a number of participants reported difficulties in developing these because of having increased workloads, a finding confirmed by Adams and Craig (2008). If resource constraints make it impossible to have multiple contacts and result in less time for home visiting, then trusting relationships between client and health visitor may not be established. If plans to increase the number of health visitors in post (Department of Health, 2011) are successful, health visitors might have more time to develop trusting relationships with clients and also to perform their wider public health role.

However, tensions are likely to remain. The White Paper Equity and Excellence (Department of Health, 2010b) has the stated aims of putting patients in charge of making decisions about their care; focussing on clinical outcomes and empowering health professionals to use their professional judgement about what is right for patients. It would seem likely therefore that the ethical tensions identified by participants in this study will continue to be experienced. Health visitors will have to continue balancing the requirements of allowing clients to exercise their own autonomy regarding their health decisions while also demonstrating improved health outcomes as defined in government policies and practicing according to their own professional judgement. It is therefore even more important that they are familiar with the ethical tensions arising between individual and public health approaches. These issues could usefully be addressed as part of the governments' stated plans to strengthen development opportunities for existing staff to restore professional autonomy and decision making (Department of Health, 2011).

\section{Strengths and limitations of the research}

Although participants were recruited on an opt-in basis from only two PCTs in England, the study benefited from the inclusion of health visitors 
who had worked among diverse populations for varying lengths of time and within different kinds of organisation arrangement. The fact that J.G. is a health visitor may have influenced decisions to take part in the study and what participants said and how during interviews. In particular, they may have been more likely to assume commonalities of understanding, but perhaps more willing to share points of personal and professional discomfort. The topic guide was used in a way that allowed participants to discuss issues that they perceived as important rather than being constrained by pre-set questions, and researchers with different disciplinary backgrounds read the interview transcripts, refined the analytic codes and discussed interpretation of data.

\section{Conclusion}

Official public health targets and the protocols that organisations develop to help achieve them are creating a number of ethical tensions for health visitors who aspire to respond to the particular needs of individual clients as well as to support the promotion of health at a population level. Although the public health ethics literature focuses mainly on this ethical tension between individual autonomy and the need for collective interventions to promote health, our research has contributed to knowledge concerning the impact that implementing collective-oriented interventions can have on the professional-client relationship. Of particular importance is the fact that current public health policies combined with resource constraints and organisational changes make the development of trusting health visitor-client relationships very difficult. These relationships influence the ability of health visitors to raise health promotion topics with their clients in ways they consider appropriate, and to support them through changes in health behaviour. The negative impact that the changes have on the health visitor-client relationship might thus mean that the way in which public health policies are being implemented may be counterproductive to the very goals that they are meant to achieve. The inability of health visitors to form trusting relationships with clients is also unsatisfactory from an ethical or professional values perspective. Government plans for the future direction of the health service seem unlikely to resolve the ethical tensions identified in this study. Policies need to be evaluated with regard to their impact upon the professional health visitor-client relationships as well as on other domains. Further attention also needs to be given to equipping practitioners to manage these ethical tensions in practice.

\section{Acknowledgements}

We would like to thank the Medical Research Council Health Services Research Collaboration for funding the project and the health visitors who took part in the research.

\section{References}

Adams, C. and Craig, I. 2008: A service at crisis point. Community Practitioner 81, 34-35.

Appleton, J.V. and Cowley, S. 2008a: Health visiting assessment - unpacking critical attributes in health visitor needs assessment practice: a case study. International Journal of Nursing Studies 45, 232-45.

Appleton, J.V. and Cowley, S. 2008b: Health visiting assessment processes under scrutiny: a case study of knowledge use during family health needs assessments. International Journal of Nursing Studies 45, 682-96.

Austin, W. 2007: The ethics of everyday practice, health care environments as moral communities. Advances in Nursing Science 30, 81-88.

Bayer, R. and Fairchild, A. 2004: The genesis of public health ethics. Bioethics 18, 473-92.

Bayer, R., Gostin, L.O., Jennings, B. and Steinbock, B. (editors) 2007: Public health ethics: theory, policy and practice. Oxford: Oxford University Press.

Beidler, S. 2005: Ethical issues experienced by communitybased nurse practitioners addressing health disparities among vulnerable populations. International Journal for Human Caring 9, 43-50.

Callahan, D. and Jennings, B. 2002: Ethics and public health: forging a strong relationship. American Journal of Public Health 92, 169-76.

Calman, K. 2009: Beyond the 'nanny state': stewardship and public health. Public Health 123 (e-Suppl), e6-10.

Childress, J.F., Faden, R.R., Gaare, R.D., Gostin, L.O., Kahn, J., Bonnie, R.J., Kass, N.E., Mastroianni, A.C., Moreno, J.D. and Nieburg, P. 2002: Public health ethics: mapping the terrain. Journal of Law, Medicine and Ethics 30, 170-78.

Clancy, A. and Svensson, T. 2007: 'Faced' with responsibility: Levinasian ethics and the challenges of responsibility in Norwegian public health nursing. Nursing Philosophy 8, 58-166. 
Cowley, S. and Frost, M. 2006: The principles of health visiting, opening the door to public health practice in the 21st century. London: CPHVA.

Cribb, A. 2005: Health and the good society: setting healthcare ethics in social context. Oxford: Oxford University Press.

Dawson, A. and Verweij, M. (editors) 2007: Ethics, prevention and public health. Oxford: Oxford University Press.

de La Cuesta, C. 1994: Relationships in health visiting: enabling and mediating. International Journal of Nursing Studies 31, 451-59.

Department of Health. 1999a: Saving lives: our healthier nation. London: The Stationery Office.

Department of Health. 1999b: Making a difference: strengthening the nursing, midwifery and health visiting contribution to health and healthcare. London: The Stationery Office.

Department of Health. 2000: The NHS plan. London: The Stationery Office.

Department of Health. 2001: Shifting the balance of power. London: The Stationery Office.

Department of Health. 2002: Liberating the talents. London: The Stationery Office.

Department of Health. 2004: Choosing health: making healthy choices easier. London: Department of Health.

Department of Health. 2006: Our health, our care, our say: a new direction for community services. London: The Stationery Office.

Department of Health. 2007: Facing the future a review of the role of health visitors. London: Department of Health.

Department of Health. 2010a: Healthy lives, healthy people: our strategy for public health in England. London: Department of Health.

Department of Health. 2010b: Equity and excellence: liberating the NHS. London: Department of Health.

Department of Health. 2011: Health visitor implementation plan 2011-15: a call to action. London: Department of Health.

Held, V. 2006: The ethics of care, personal, political and global. Oxford: Oxford University Press.

Holland, S. 2007: Public health ethics. Cambridge: Polity Press.

Kass, N.E. 2001: An ethics framework for public health. American Journal of Public Health 91, 1776-82.
Lacey, J. 2009: Alcohol brief interventions: exploring perceptions and training needs. Community Practitioner $82,30-33$.

Lakshman, R., Ogilvie, D. and Ong, K.K. 2009: Mothers experiences of bottle-feeding: a systematic review of qualitative and quantitative studies. Archives of Disease in Childhood 94, 596-601.

McNaughton, D. 2000: A synthesis of qualitative home visiting research. Public Health Nursing 17, 405-14.

Moules, N.J., MacLeod, M.L.P., Thirsk, L.M. and Hanlon, N. 2010: "And then you'll see her in the grocery store": the working relationships of public health nurses and highpriority families in northern Canadian Communities. Journal of Paediatric Nursing 25, 327-34.

Nuffield Council on Bioethics. 2007: Public health: ethical issues. Cambridge: Cambridge Publishers Ltd.

Nursing and Midwifery Council (NMC). 2007: The code: standards of conduct, performance and ethics for nurses and midwives. London: NMC.

Ritchie, J. and Spencer, L. 1994: Qualitative data analysis for applied policy research. In Bryman, A. and Burgess, R.G., editors, Analysing qualitative data. London: Routledge, 173-94.

Rogers, W.A. 2002: Is there a tension between doctors' duty of care and evidence-based medicine? Health Care Analysis 10, 277-87.

Rogers, W.A. 2004: Evidence based medicine and justice: a framework for looking at the impact of EBM upon vulnerable or disadvantaged groups. Journal of Medical Ethics 30, 141-45.

Russell, S. 2008: Left fending for ourselves: a report on the health visiting service as experienced by mums. Watford: Netmums.

Tannahill, A. 2008: Beyond evidence-to ethics: a decisionmaking framework for health promotion, public health and health improvement. Health Promotion International 23, 380-90.

Worth, A. and Hogg, R. 2000: A qualitative evaluation of the effectiveness of health visiting practice. British Journal of Community Nursing 5, 221-22. 